\title{
Gastro-oesophageal reflux and aspiration in patients with advanced lung disease
}

\author{
M P Sweet, ${ }^{1}$ M G Patti, ${ }^{1}$ C Hoopes, ${ }^{1}$ S R Hays, ${ }^{2}$ J A Golden ${ }^{2}$
}

${ }^{1}$ University of California San Francisco, San Francisco, California, USA; ${ }^{2}$ Departments of Surgery and Medicine, University of California San Francisco, San Francisco, California, USA

Correspondence to: Dr C Hoopes, Department of Surgery, University of California San Francisco, 500 Parnassus Ave, MU W405, Box 0118, San Francisco, CA 94117, USA; hoopesc@surgery.ucsf.edu

Received 12 April 2007 Accepted 29 June 2007

\begin{abstract}
Numerous small observational studies have shown that gastro-oesophageal reflux is prevalent among patients with advanced lung disease. The fundamental concern is that reflux is a risk factor for recurrent microaspiration, which may cause lung injury. For example, in lung transplant patients, a molecular marker of aspiration was a risk factor for the bronchiolitis obliterans syndrome in one study. To date, however, there are no large prospective studies measuring the impact of aspiration on clinical outcomes. The major obstacle limiting the study of reflux and aspiration in patients with advanced lung disease is the absence of a reliable diagnostic tool. Proximal oesophageal acid detection by $\mathrm{pH}$ monitoring is the only widely available measure of aspiration risk. Impedance monitoring may be a superior measure of aspiration risk as it measures both acid and non-acid reflux episodes. Molecular markers of aspiration, such as pepsin or bile salts in the bronchoalveolar lavage or exhaled breath condensate, may be the optimal diagnostic tests, but they are not currently available outside the research setting. Larger observational studies are needed to determine the following: (1) the clinical significance of aspiration in patients with advanced lung disease and in patients who have had lung transplantation and (2) the diagnostic test that best predicts adverse outcomes.
\end{abstract}

Gastro-oesophageal reflux disease (GORD) is recognised as an aetiology of cough and aspiration related acute pulmonary injury. ${ }^{12}$ The association between reflux, microaspiration and chronic lung injury is not well characterised. The reason this subject has generated so much interest despite the relative paucity of good outcome data is because GORD and aspiration are potentially treatable.

This review describes the association between gastro-oesophageal reflux and aspiration among adults with advanced lung disease (ALD) and among those who have had lung transplantation. The associations between reflux and asthma, laryngopharyngeal reflux and cough have been reviewed elsewhere. ${ }^{3}$ This review will also address the issue of diagnostic testing and suggest directions for future studies.

\section{GASTR0-0ESOPHAGEAL REFLUX AND LUNG DISEASE}

\section{Historical perspective}

In 1949, Belcher summarised a number of case series published in the first half of the 20th century. ${ }^{4}$ He credited Vinson with the first report of a lung abscess caused by aspiration in a patient with achalasia in 1927. Several studies performed in the 1960s and 70s reported that pulmonary fibrosis was common among patients with a radiographic or clinical diagnosis of reflux..$^{5-7}$

Pellegrini et al published a landmark paper in 1979 that helped establish several key principles of extraoesophageal complications of GORD. ${ }^{8}$ Among 100 patients with pathological reflux on $\mathrm{pH}$ monitoring, those with weak oesophageal peristalsis and slow oesophageal clearance were more likely to have respiratory symptoms immediately following reflux events. Therefore, without adequate oesophageal motility, reflux events were more likely to cause respiratory symptoms. Furthermore, oesophageal $\mathrm{pH}$ testing clarified the temporal association between reflux and cough. Some patients had reflux events following cough, presumably because of increased intra-abdominal pressures caused by coughing. Other patients had cough immediately following a reflux event, suggesting that reflux induced the cough. The authors also found that typical reflux symptoms (heartburn, regurgitation and dysphagia) had limited correlation with objectively measured reflux.

The first published report of an association between oesophageal dysfunction and parenchymal lung disease is attributed to Denis and colleagues. ${ }^{9}$ In 1981, they reported that 16 of 24 patients $(67 \%)$ with systemic sclerosis had impaired oesophageal motility that was associated with decreased lung compliance. ${ }^{9}$ In 1989, Johnson et al studied oesophageal function and aspiration in a cohort of 13 patients with systemic sclerosis. ${ }^{10}$ Abnormal reflux and laryngoscopic examination findings suggestive of laryngopharyngeal reflux were common. A multivariate regression model found an association between reflux and impaired diffusing capacity.

In addition to showing that reflux was associated with lung fibrosis, Johnson and colleagues ${ }^{10}$ made the important contribution of measuring proximal oesophageal acid exposure with a dual sensor pH monitor. In 1993, Patti et al used a similar dual sensor $\mathrm{pH}$ monitor to correlate cough with proximal oesophageal reflux. ${ }^{11}$ They speculated that when cough was temporally associated with distal reflux only, then the symptoms were attributed to a vagal reflux arc from oesophageal irritation. When cough was associated with reflux that extended into the proximal oesophagus, then microaspiration was the likely aetiology of the cough. ${ }^{12}$ Furthermore, proximal oesophageal reflux was more common among those patients with motility abnormalities of the lower oesophageal sphincter and oesophageal body.

Until 1996, data on the association between reflux and respiratory complications were limited 
to single institutional case series. El-Serag and Sonnenberg confirmed the findings of these case series in a population setting. ${ }^{13}$ Patients with erosive oesophagitis, a sign of significant GORD, had a 1.36 odds ratio (OR) of pulmonary fibrosis, a 1.28 OR of chronic bronchitis and a 1.22 OR of chronic obstructive pulmonary disease (COPD) in a case control study of more than 200000 US veterans. Although these data are not proof of causation, they confirm the presence of an association between GORD and ALD in a large population based setting.

As described below, GORD is prevalent among patients with diverse forms of ALD. The pathophysiology of this association is not well characterised. For several decades, oesophageal specialists have postulated that changes in diaphragmatic position (with compromise of the diaphragmatic pinch-cock action) and increases in positive intra-abdominal pressure and negative intrathoracic pressure (with a corresponding increase in the pressure gradient) have facilitated reflux. These hypotheses have never been proved.

\section{GASTRO-0ESOPHAGEAL REFLUX AND SPECIFIC LUNG DISEASES}

\section{Idiopathic pulmonary fibrosis/usual interstitial pneumonia}

While the pathophysiology of idiopathic pulmonary fibrosis/ usual interstitial pneumonia (IPF) appears to involve aberrant fibroblast proliferation as a result of recurrent epithelial injury, the aetiology is unknown. ${ }^{14}$ Speculation has arisen that reflux may play a role in the pathogenesis and/or progression of IPF (specifically in acute exacerbation of IPF) as a recurrent inflammatory stimulus. ${ }^{15}$ Furthermore, reflux plays a role in symptoms often attributed to interstitial lung disease, of which IPF is the most prevalent form. ${ }^{16}$

Four studies have found a high prevalence of reflux among patients with IPF (table 1). ${ }^{15}$ 17-19 The largest of these, by Raghu and colleagues, ${ }^{17}$ used heterogeneous oesophageal testing and tested some patients while they were taking proton pump inhibitors (PPIs). These limitations prevent wide generalisation of their data. That a consistently high prevalence has been seen across all studies, however, suggests that the association is valid.

The clinical impact of reflux on IPF disease progression has not been thoroughly examined. A case series by Linden and colleagues demonstrated that 11 pre-transplant patients with IPF undergoing antireflux surgery had reduced supplementary oxygen dependence compared with other pre-transplant patients with IPF after a median of 11 months of follow-up. ${ }^{20}$ Other more objective measures of pulmonary function, (eg, walk test and pulmonary function tests) however, were not improved after surgery. Raghu et al reported a case series of four patients with IPF and a clinical diagnosis (ie, symptoms) of reflux. $^{21}$ Two patients had deterioration in their pulmonary function tests that resolved with control of oesophageal acid exposure. This small study was the first to temporally correlate IPF clinical stability with control of acid reflux. Furthermore, there are anecdotal cases of IPF disease stability following treatment for reflux. ${ }^{20}$ While it cannot be proven that disease stability was caused by control of reflux, they do offer hope that a subset of patients with IPF may benefit from antireflux therapy.

\section{Cystic fibrosis}

While there are few data regarding GORD in adults with cystic fibrosis (CF), small cohort studies have consistently found a high prevalence of abnormal reflux (table 1) ${ }^{22-25}$ In one study of 50 adults with CF, 47 (94\%) reported symptoms of reflux and eight of 10 studied by $\mathrm{pH}$ monitoring had pathological reflux. ${ }^{22}$ The same authors then found that tracheal acidification followed oesophageal acidification in four of the eight patients with pathological reflux, all of whom had DeMeester scores $\geqslant 60 .{ }^{26}$ Gastroparesis, seen in 20 of 30 pre-transplant patients in another study, may be a significant contributing factor for reflux in patients with $\mathrm{CF} .{ }^{27}$ Most adults with $\mathrm{CF}$, therefore, have GORD, and perhaps a broader disorder of foregut motility.

\section{Connective tissue disease}

Systemic sclerosis often involves the oesophagus, causing profound impairment of oesophageal motility and severe reflux. A cohort study of 47 patients with polymyositis or dematomyositis and lung disease found that 18 (47\%) had dysphagia, six $(14 \%)$ had aspiration pneumonia, and oesophageal motility abnormalities were found by oesophagram or oesophageal motility studies in eight of 27 patients (30\%). ${ }^{28}$ Among a cohort of 125 patients with scleroderma (51 (41\%) of whom had pulmonary involvement), 45 (36\%) had oesophagitis and 61 of the 78 (78\%) who had oesophageal pH measured had pathological GORD. ${ }^{29}$ These data were confirmed by an ongoing study at our institution that has found that 18 of 23 patients (83\%) referred for lung transplantation with connective tissue diseases (ie, scleroderma, mixed connective tissue disease or dematomyositis) had pathological GORD, and 18 (78\%) with manometry data had aperistalsis or abnormal peristalsis (unpublished data). Again, in this subgroup with ALD, GORD and foregut dysmotility were common.

\section{Obstructive lung disease}

GORD is also prevalent among patients with COPD, although less so than in patients with fibrotic lung disease. A cross sectional study of 512 people with bronchial asthma or chronic bronchitis found an overall prevalence of benign oesophageal disease of $44.5 \%$ compared with $34.5 \%$ of patients without known lung disease..$^{30}$ Oesophageal $\mathrm{pH}$ testing again confirmed that reflux was prevalent (table 1). ${ }^{25} 3031$

Reflux has also been associated with COPD using population based data. In a large VA study, El-Serag and Sonnenberg found that reflux oesophagitis was associated with COPD, with an OR of 1.22 (95\% confidence interval (CI) 1.16 to 1.27$).{ }^{13}$ Another population based case control study found that COPD was a risk factor for a diagnosis of GORD, with an OR of 1.3 (95\% CI 1.0 to 1.8$){ }^{32}$

Little is known about the link between GORD and outcomes in patients with COPD. Using a cross sectional sample, RasconAguilar found an association between the presence of GORD symptoms and increased frequency of COPD exacerbations. ${ }^{33}$ Another cross sectional study found that decreases in oesophageal $\mathrm{pH}$ corresponded with drops in oxygen saturation among six of 15 patients with GORD and COPD. ${ }^{31}$

\section{Lung transplantation}

The most active area of the study of microaspiration is taking place in lung transplant patients. Survival following lung transplantation is inferior to that of other solid organ transplantation, with median survival less than 5 years. Obliterative bronchiolitis, characterised by its clinical correlate the bronchiolitis obliterans syndrome (BOS), is the main complication limiting allograft survival. ${ }^{34}$ Reflux and microaspiration have been shown to be risk factors for BOS following transplant. 
Table 1 Prevalences of abnormal gastro-oesophageal reflux (pH testing) and of delayed gastric emptying (nuclear medicine study)

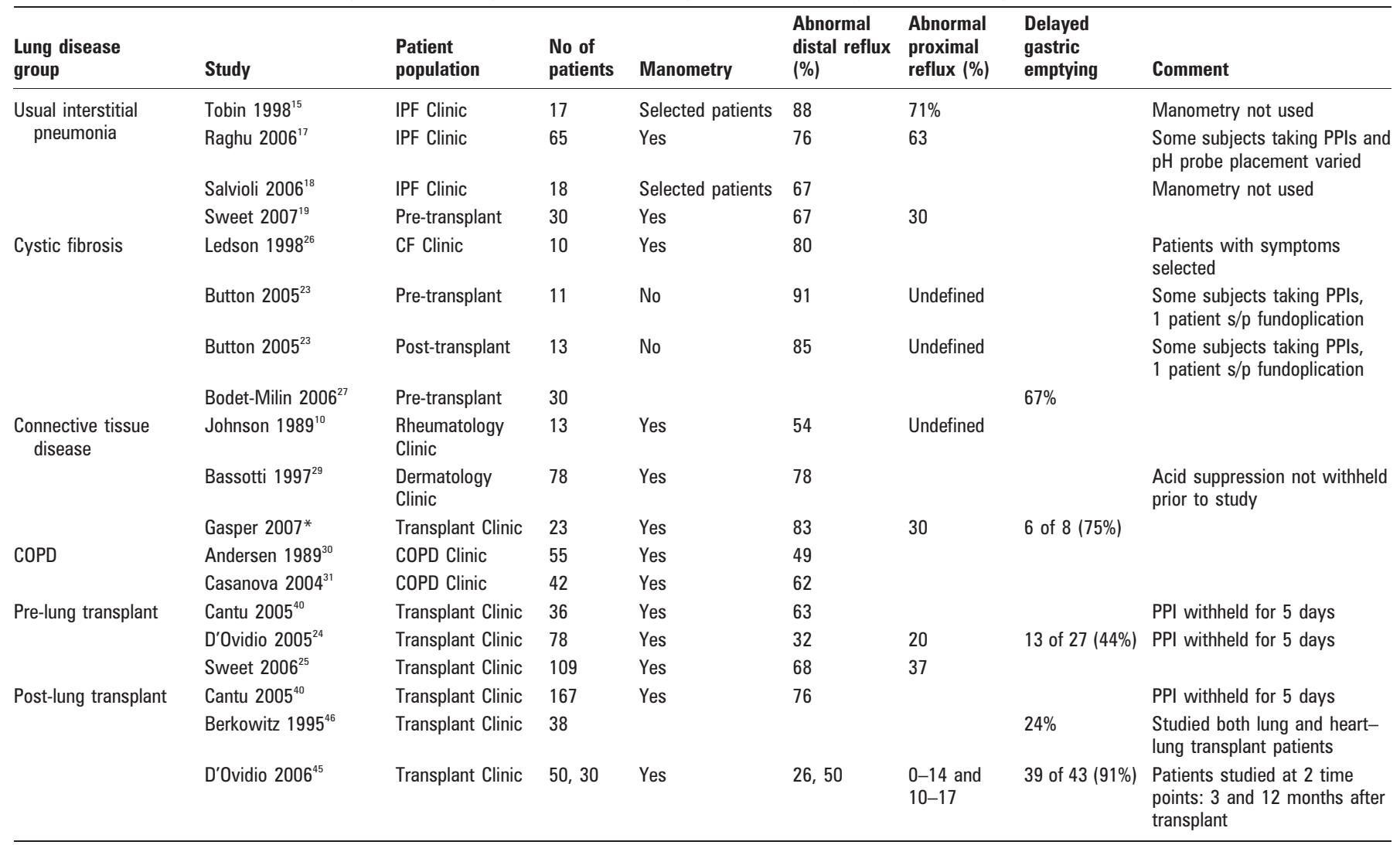

\footnotetext{
*Unpublished data.
}

COPD, chronic obstructive pulmonary disease; IPF, idiopathic pulmonary fibrosis; PPIs, proton pump inhibitors; s/p, status post.

In the 1990s, several small case series were published describing foregut dysfunction among lung and heart-lung transplant patients. ${ }^{35-39}$ Subsequently, the lung transplantation team at Duke University initiated the first systematic and objective assessment of reflux in lung transplant patients. They measured distal oesophageal acid exposure in over 200 patients and found prevalences of $63 \%$ and $76 \%$ in the pre and posttransplant subgroups, respectively (table 1)..$^{40}$

The group began performing antireflux surgery after observing that reflux was associated with declines in lung function. ${ }^{41}{ }^{42}$ They reported that the BOS resolved in a few patients following fundoplication. An outcome analysis of 14 patients who had antireflux surgery within 1 month of their transplant reported $100 \%$ freedom from BOS at 1 and 3 years after transplant. ${ }^{40}$ Only five patients, however, were followed-up to the 3 year time point. In this retrospective study, there was no difference in the incidence of BOS among those patients with medically managed reflux and those without reflux. If the hypothesis that aspiration is a risk factor for BOS is true, then we would expect to have seen a difference between these groups. Medical therapy normalises the $\mathrm{pH}$ of the refluxate, but does not stop the mechanical reflux process. ${ }^{43}$ The pathogenesis of BOS is likely multifactorial, and this study did not adjust for clinical covariates. Furthermore, proximal reflux is a better predictor of aspiration risk and this study relied on distal reflux measurements only. Again, despite their limitations, these encouraging initial results support further study of this association.

Two other cross sectional cohort studies of pre-transplant patients have found high prevalences of GORD (table 1). ${ }^{24} 25$ D'Ovidio et al found a lower prevalence than the other groups, and this may be due to the fact that PPIs were withheld for only 5 days prior to the $\mathrm{pH}$ studies. Abnormal oesophageal motility was seen in $33 \%$ and $55 \%$ of the two cohorts, and delayed gastric emptying was seen in 13 of 27 (44\%) of patients in one study.

\section{Effect of transplant on gastro-oesophageal reflux}

Lung transplant appears to worsen oesophageal reflux. Among 23 patients studied, both before and after transplant, the prevalence of abnormal distal reflux rose from $35 \%$ to $65 \% .{ }^{44}$ Another study found that the prevalence of pathological reflux increased from $32 \%$ at 3 months after transplant to $53 \%$ at 12 months. ${ }^{45}$ This is likely because of complications of the transplantation. Vagal nerve injury is a common complication of heart or lung transplantation. Gastroparesis as a result of vagal nerve injury and/or immunosuppression may play a role in the development of reflux after transplantation. Gastric motility is frequently abnormal in heart or lung transplant patients. ${ }^{35}$ Berkowitz et al studied 38 post-thoracic transplant patients, $24 \%$ of whom had delayed gastric emptying (table 1). ${ }^{46}$ Furthermore, chronic allograft dysfunction was more frequent among patients with delayed gastric emptying. Subsequent studies of patients with lung disease have found that delayed gastric emptying was prevalent. ${ }^{25}{ }^{44}$ D'Ovidio et al reported that $91 \%$ of gastric emptying studies were abnormal among a cohort of 43 post-transplant patients. ${ }^{45}$ The prevalence may be higher among some high risk populations. For instance, 29 of 30 patients (97\%) with CF had abnormal gastric emptying following lung or heart-lung transplantation in one study. ${ }^{27}$ 


\section{ASPIRATION}

Although abnormal reflux is prevalent among patients with ALD, we do not know who among those with reflux are aspirating. Several studies have attempted to determine the prevalence of aspiration after lung transplantation. D'Ovidio et al examined bile salts in the bronchoalveolar lavage (BAL) of 120 post-transplant patients in a cross sectional study. ${ }^{47}$ The authors found a $17 \%$ prevalence of elevated concentrations of bile salts. Furthermore, the highest concentrations were found in patients with early onset BOS ( $<1$ year post-transplant). The study reported mean concentrations of bile salts among those with early BOS, late BOS and no BOS. There was a trend for decreasing concentrations across the groups, suggesting a dose effect association between elevated BAL bile salt concentration and the time to development of BOS. Furthermore, the authors found that elevated BAL bile acids were associated with alveolar neutrophilia, interleukin 8 and cultures positive for bacteria and fungus.

The same group subsequently showed that the presence of bile salts in the BAL fluid at 3 months after transplant was associated with the development of BOS in a time and dose dependent manner. ${ }^{45}$ This important study was the first to prospectively evaluate post-transplant patients and show that markers of aspiration are a risk factor for BOS. The prevalence of aspiration, as measured by bile salts, was $43 \%$ at 3 months after transplant. The presence of bile salts in the BAL was also associated with decreased surfactant collectin proteins and surfactant phospholipids. Bile salts may, therefore, cause lung injury by impairing the innate immunity of the allograft. ${ }^{48}$ The limitations of this study are the lack of adjustment for covariates and the small cohort size of 37 . The study demonstrates a dose effect and temporal association, two important indicators of causality.

Measurement of bile salts may underestimate the prevalence of aspiration, as only $58 \%$ of patients without GORD and $75-$ $86 \%$ of those with GORD will have duodeno-gastro-oesophageal reflux with bile in the refluxate. ${ }^{49-51}$ Specific markers of gastric fluid in BAL may prove more sensitive than bile salts as a screening test of aspiration. A small cross sectional study by Ward et al found pepsin, a gastric protease, in the BAL fluid of 13 post-transplant subjects. ${ }^{52}$ No pepsin was detected in the BAL specimens from four control subjects. Another small cohort study by Farrell et al correlated proximal oesophageal reflux with BAL pepsin levels in 33 children with reflux. ${ }^{53}$ Again, no pepsin was identified in the BAL fluid of 13 negative control patients. Stovold et al recently reported on a cross sectional study comparing BAL pepsin with findings of acute rejection in 36 post-transplant patients. BAL pepsin concentrations were elevated in seven of 12 post-transplant patients with acute perivascular (A2) rejection. ${ }^{54}$

The main limitation of using molecular markers of aspiration in BAL samples is the inability to standardise the concentration. It is not known how BAL pepsin or bile salt concentrations change over time following an aspiration event. Elevated levels may reflect less dilution at the time of sampling, high volume and/or high frequency aspiration events, or impaired clearance by the lung itself.

Hartwig et al recently published a study describing a rat lung transplant model of aspiration. They found that exposure of the lung to filtered gastric aspirate induced fibrotic changes and severe acute rejection. ${ }^{55}$ This model may be useful in elucidating the immunological covariates that affect the lung's response to aspiration.
The specific elements of the aspirate that are most injurious remain unknown. Refluxate $\mathrm{pH}$ varies, as virtually all posttransplant patients and many patients with ALD are treated with acid suppressing drugs in conjunction with steroids. Duodenal reflux of bile salts and reflux of other gastric contents (eg, the protease pepsin as well as infectious pathogens) may be important contributing factors to lung injury caused by aspiration. Which element is most injurious has important therapeutic implications. Acid suppression can be achieved pharmacologically, but the mechanical reflux events themselves may require additional treatment in the form of prokinetic medications or surgical fundoplication. Acid suppression therapy also changes the flora of the gastric refluxate. ${ }^{56} 57$

Patients with lung disease are particularly vulnerable to aspiration events. Aspiration of nasopharyngeal contents may occur in half of otherwise healthy adults, as measured by pulmonary scintigraphy. ${ }^{58}$ Such events are controlled by normal cough reflex, mucociliary clearance and a functional immune system. ${ }^{2}$ Lung transplant patients have impairment of all three defences. ${ }^{59}{ }^{60}$ Patients with IPF and other forms of ALD are also less likely to tolerate aspiration events than are healthy people, although there are no specific data addressing this subject.

\section{OUTCOME}

Thus far, outcome data demonstrating a causal association between reflux mediated microaspiration and chronic lung injury are limited to the single observational study of 37 posttransplant patients discussed above. The dose effect and temporal association between BAL bile salt concentration and time to onset of BOS is strong evidence of a link between these two processes. ${ }^{45}$ Among the five patients with high levels of bile salts in the BAL fluid, the incidence of BOS at 30 months was $80 \%$, four times the incidence among patients without detectable bile salts. These results must be confirmed in larger cohorts to better understand the magnitude of the effect and what covariates influence the process. At this time, there are no prospective outcome data demonstrating that reflux or aspiration are harmful in patients with ALD.

\section{DIAGNOSTIC TESTING: THE CURRENT LIMITING FACTOR}

A fundamental issue limiting the further study of GORD related microaspiration is the lack of a gold standard diagnostic test. Clinicians use multiple different tools to screen for and diagnose reflux. The general diagnostic approach to GORD and the approach for extraoesophageal symptoms of GORD have been reviewed elsewhere. ${ }^{3}$ There are, however, some specific data regarding diagnosis in patients with ALD.

Typical reflux symptoms of heartburn, regurgitation and dysphagia are of no use when screening for reflux in patients with ALD. A cross sectional study using a constellation of "gold standards" (ie, abnormality of oesophageal manometry, oesophageal $\mathrm{pH}$ or endoscopy) for diagnosis of GORD found that symptoms had a sensitivity and specificity of $89.5 \%$ and $47.1 \%$ among 512 people with asthma or chronic bronchitis. ${ }^{30}$ When the presence of any typical reflux symptom (heartburn, regurgitation and/or dysphagia) was compared with objective findings at $\mathrm{pH}$ study in 109 patients with various types of ALD, the sensitivity, specificity and likelihood ratio positive were $67 \%, 26 \%$ and 0.91 , respectively. ${ }^{25}$ Similar findings of low sensitivity and specificity of reflux symptoms have been found in cohort studies of patients with COPD, CF and IPF. ${ }^{17} 23$ 31 In a cohort of 518 patients, Oelschlager et al showed that typical reflux symptoms did not discriminate among patients with and 
without pharyngeal acid exposure. ${ }^{61}$ Reflux is often silent in patients with ALD, and symptoms of heartburn and regurgitation are common among patients with normal objective measures of reflux.

Oesophageal $\mathrm{pH}$ monitoring is the most widely available test, although important limitations exist. Dual sensor $\mathrm{pH}$ monitoring is essential as it identifies the subgroup of patients with abnormal proximal reflux. Among a cohort of 518 patients with symptoms referred for oesophageal studies, 43 of 181 (24\%) patients with abnormal acid reflux extending into the pharynx had normal distal oesophageal acid exposure. ${ }^{61}$ In these patients, the frequency and duration of reflux events was within physiological norms for the distal oesophagus, but so many of these events extended upward into the proximal oesophagus that they exceeded the normal standards for proximal reflux. Temporal correlation between proximal reflux events and respiratory symptoms of cough is a strong predictor of who is most likely to benefit from antireflux therapy. ${ }^{12}$ It may not be possible to measure this correlation in patients with ALD given the frequency and multiple aetiologies of cough in these patients. Potluri et al found that proximal reflux correlated with salivary pepsin concentration, indicating that proximal oesophageal reflux events frequently extend into the hypopharynx. ${ }^{62}$ Most recently, two studies have shown that proximal reflux correlates with elevated concentrations of BAL pepsin and bile salts, two markers of aspiration. ${ }^{45}$ The other advantage of oesophageal testing is the assessment of oesophageal motor function. As discussed above, oesophageal peristalsis is one of the key protective measures against aspiration and it is frequently abnormal in patients with ALD. The limitation of $\mathrm{pH}$ testing relates to the use of gastric acid as a surrogate marker of reflux events. This requires cessation of PPIs. Furthermore, $\mathrm{pH}$ testing will not detect neutral or mildly acidic reflux events, which are common. ${ }^{63} 64$

The ability of $\mathrm{pH}$ studies to predict aspiration risk is not well characterised, as only two studies have compared oesophageal $\mathrm{pH}$ measurement with other diagnostic tools. D'Ovidio et al found that $75 \%$ of patients with high levels of bile salt in the BAL had abnormal proximal oesophageal $\mathrm{pH}$ studies, meaning that $25 \%$ of patients with the highest quantity of aspiration had normal proximal oesophageal $\mathrm{pH}$ measurements. In contrast, $15 \%$ of patients without measurable bile salts had abnormal proximal oesophageal $\mathrm{pH}$ measurements. ${ }^{45}$ Therefore, proximal oesophageal $\mathrm{pH}$ testing had a sensitivity of $75 \%$, specificity of $81 \%$ and a likelihood ratio of 4 for detecting aspiration of high levels of bile salts. For any bile salt aspiration, values are $45 \%$, $85 \%$ and 3 , respectively. A study in a heterogenous cohort of 51 children with unexplained respiratory symptoms (cough, pneumonia, apnoea, asthma and laryngitis) found that aspiration, as measured by pulmonary scintigraphy, was found in $49 \%$. Oesophageal pH studies were not helpful in the diagnosis of aspiration in this study, with a sensitivity of $24 \%$, specificity of $65 \%$ and positive predictive value of $46 \% .{ }^{65}$ These studies highlight the challenges facing clinicians and investigators. The test characteristics of BAL bile salts and of pulmonary scintigraphy are not well known, and without that information, they cannot be used as the gold standard against which to compare $\mathrm{pH}$ studies. Furthermore, all of these tests measure a brief time period, and as such may not reflect the longer term steady state. To resolve these discrepancies between diagnostic methodologies, studies should compare which test is most strongly predictive of adverse outcomes. In the D'Ovidio study, both $\mathrm{pH}$ testing and BAL bile salts were predictive of $\mathrm{BOS}$, but both results were dichotomised into normal and abnormal, reducing the precision of the measurements. ${ }^{45}$

Oesophageal impedance testing is a new technology that discriminates gas and fluid reflux regardless of $\mathrm{pH} .{ }^{64}$ One study elegantly demonstrated this when the investigators measured the number and magnitude of reflux events in the same patients on and off PPI therapy. There was no change in the number or magnitude of events; the only change was the $\mathrm{pH}$ of the refluxate. ${ }^{43}$ Impedance can be used to diagnose GORD regardless of the use of acid blocking medications. ${ }^{66}$ Impedance is particularly valuable when assessing aspiration risk, as aspiration is a mechanical process, and the $\mathrm{pH}$ of the refluxate is not the specific concern. Tutuian et al demonstrated that impedance testing discriminated the subgroup of 13 patients $(26 \%$ of the cohort) who had persistent cough following reflux events despite PPI therapy. ${ }^{67}$ Another study found that patients with reflux symptoms and normal $\mathrm{pH}$ tests were more likely to have non-acid reflux episodes extending into the proximal oesophagus than were control patients. ${ }^{63}$ There are no studies describing impedance measures in patients with ALD. Furthermore, as is true of $\mathrm{pH}$ testing, impedance can only measure aspiration risk.

Another new diagnostic test currently being studied is exhaled breath condensate (EBC). EBC may provide a rapid, reproducible, inexpensive and non-invasive way to sample the airway for markers of aspiration. EBC $\mathrm{pH}$ decreases following acid aspiration. ${ }^{68}$ Low EBC $\mathrm{pH}$ has also been associated with BOS and acute rejection after lung transplantation. ${ }^{69}$ Recently, pepsin has been identified in EBC fluid from post-transplant patients. $^{70} \mathrm{EBC} \mathrm{pH}$ or pepsin may be useful as an initial screening test to select those patients at highest risk for further invasive testing.

Biochemical markers of aspiration, as discussed above, will likely prove to be the best diagnostic test. Currently, few patients have been studied, and it may be difficult to establish normal standards for these tests given an inability to standardise concentration.

Although $\mathrm{pH}$ studies have their limitations (eg, operator dependence, patient discomfort, and imperfect sensitivity and specificity for aspiration), proximal oesophageal reflux is the only clinically available surrogate marker of aspiration risk. Until impedance testing and markers of aspiration have been more carefully studied, oesophageal $\mathrm{pH}$ testing remains the only widely available diagnostic tool.

\section{FUTURE DIRECTIONS}

Future studies should be directed to answer the following two questions: (1) are reflux and microaspiration associated with adverse clinical outcomes in patients with ALD and, if so, (2) which diagnostic test best predicts those outcomes. As the majority of data currently available come from cross sectional studies, it remains unknown whether the association between reflux and ALD is causal. Furthermore, thoracic mechanical changes caused by ALD may in fact cause or worsen reflux. Therefore, the first task is to confirm that reflux and aspiration are causally associated with worse clinical outcomes. Such data are needed to develop evidence based screening protocols and to support further studies addressing pathophysiology and therapy.

Patients with ALD and those referred for transplantation are complex. Immunological risk, environmental exposure and other medical comorbidities can differ substantially among these patients. It will require systematic evaluation to identify and isolate the risk factors that play a role in this association. It is unlikely that any single institution will be able to recruit a 
sufficient cohort to resolve these issues, and therefore multicentre studies are needed.

\section{CONCLUSIONS}

GORD and aspiration are common and often severe among patients with ALD. Following lung transplantation, both GORD and aspiration appear to be risk factors for the development of BOS. Larger prospective studies are needed to clarify the clinical impact of reflux and aspiration on clinical outcomes. These issues are of tremendous clinical importance because GORD and aspiration are modifiable. A better understanding of aspiration in ALD may provide new means of treating these otherwise challenging diseases.

Competing interests: None.

\section{REFERENCES}

1. Irwin RS. Chronic cough due to gastroesophageal reflux disease: ACCP evidencebased clinical practice guidelines. Chest 2006;129(1 Suppl):80S-94.

2. Marik PE. Aspiration pneumonitis and aspiration pneumonia. N Engl J Med 2001;344:665-71.

3. Napierkowski J, Wong RK. Extraesophageal manifestations of GERD. Am J Med Sci 2003:326:285-99.

4. Belcher JR. The pulmonary complications of dysphagia. Thorax 1949;4:44-56.

5. Mays EE, Dubois JJ, Hamilton GB. Pulmonary fibrosis associated with tracheobronchial aspiration. A study of the frequency of hiatal hernia and gastroesophageal reflux in interstitial pulmonary fibrosis of obscure etiology. Chest 1976;69:512-15.

6. Pearson JEG, Wilson RSE. Diffuse pulmonary fibrosis and hiatus hernia. Thorax 1971;26:300-5

7. Urschel HC Jr, Paulson DL. Gastroesophageal reflux and hiatal hernia. Complications and therapy. J Thorac Cardiovasc Surg 1967;53:21-32.

8. Pellegrini CA, DeMeester TR, Johnson LF, et al. Gastroesophageal reflux and pulmonary aspiration: incidence, functional abnormality, and results of surgical therapy. Surgery 1979;86:110-19.

9. Denis $\mathbf{P}$, Ducrotte $\mathbf{P}$, Pasquis $\mathbf{P}$, et al. Esophageal motility and pulmonary function in progressive systemic sclerosis. Respiration 1981:42:21-4.

10. Johnson DA, Drane WE, Curran J, et al. Pulmonary disease in progressive systemic sclerosis. Arch Intern Med 1989:149:589-93.

11. Patti MG, Debas HT, Pellegrini CA. Clinical and functional characterization of high gastroesophageal reflux. Am J Surg 1993;165:163-8.

12. Patti MG, Arcerito M, Tamburini A, et al. Effect of laparoscopic fundoplication on gastroesophageal reflux disease-induced respiratory symptoms. J Gastrointest Surg 2000:4:143-9.

13. El-Serag H, Sonnenberg A. Comorbid occurrence of laryngeal or pulmonary disease with esophagitis in United States military veterans. Gastroenterology 1997:113:755-60.

14. Harari S, Caminati A. Idiopathic pulmonary fibrosis. Allergy 2005:60:421-35.

15. Tobin RW, Pope CE 2nd, Pellegrini CA, et al. Increased prevalence of gastroesophageal reflux in patients with idiopathic pulmonary fibrosis. Am J Respir Crit Care Med 1998;158:1804-8.

16. Madison JM, Irwin RS. Chronic cough in adults with interstitial lung disease. Curr Opin Pulm Med 2005:11:412-16.

17. Raghu G, Freudenberger TD, Yang S, et al. High prevalence of abnormal acid gastrooesophageal reflux in idiopathic pulmonary fibrosis. Eur Respir J 2006;27:136-42.

18. Salvioli B, Belmonte G, Stanghellini V, et al. Gastro-oesophageal reflux and interstitial lung disease. Dig Liver Dis 2006;38:879-84.

19. Sweet MP, Patti MG, Leard LE, et al. Gastroesophageal reflux in patients with idiopathic pulmonary fibrosis referred for lung transplantation. J Thorac Cardiovasc Surg 2007;133:1078-84.

20. Linden PA, Gilbert RJ, Yeap BY, et al. Laparoscopic fundoplication in patients with end-stage lung disease awaiting transplantation. J Thorac Cardiovasc Surg 2006;131:438-46.

21. Raghu G, Yang ST, Spada C, et al. Sole treatment of acid gastroesophageal reflux in idiopathic pulmonary fibrosis: a case series. Chest 2006;129:794-800.

22. Ledson MJ, Tran J, Walshaw MJ. Prevalence and mechanisms of gastrooesophageal reflux in adult cystic fibrosis patients. J $R$ Soc Med 1998:91:7-9.

23. Button B, Roberts $S$, Kotsimbos $T$, et al. Gastroesophageal reflux: a potentially significant problem in patients with cystic fibrosis before and after lung transplantation. J Heart Lung Transplant 2005;24:1522-9.

24. D'Ovidio F, Singer LG, Hadjiliadis D, et al. Prevalence of gastroesophageal reflux in end-stage lung disease candidates for lung transplant. Ann Thorac Surg 2005;80:1254-60.

25. Sweet MP, Herbella FAM, Leard L, et al. The prevalence of distal and proximal gastroesophageal reflux in patients awaiting lung transplantation. Ann Surg 2006;244:491-7

26. Ledson MJ, Wilson GE, Tran J, et al. Tracheal microaspiration in adult cystic fibrosis. $J R$ Soc Med 1998:91:10-12.
27. Bodet-Milin C, Querellou S, Oudoux A, et al. Delayed gastric emptying scintigraphy in cystic fibrosis patients before and after lung transplantation. J Heart Lung Transplant 2006;25:1077-83.

28. Dickey BF, Myers AR. Pulmonary disease in polymyositis/dermatomyositis. Semin Arthritis Rheum 1984;14:60-76.

29. Bassotti G, Battaglia E, Debernardi V, et al. Esophageal dysfunction in scleroderma: relationship with disease subsets. Arthritis Rheum 1997:40:2252-9.

30. Andersen LI, Jensen G. Prevalence of benign oesophageal disease in the Danish population with special reference to pulmonary disease. J Intern Med 1989;225:393-402.

31. Casanova C, Baudet JS, del Valle Velasco M, et al. Increased gastro-oesophageal reflux disease in patients with severe COPD. Eur Respir J 2004;23:841-5.

32. Ruigomez A, Garcia Rodriguez LA, Wallander MA, et al. Natural history of gastrooesophageal reflux disease diagnosed in general practice. Aliment Pharmacol Ther 2004;20:751-60.

33. Rascon-Aguilar IE, Pamer M, Wludyka $\mathrm{P}$, et al. Role of gastroesophageal reflux symptoms in exacerbations of COPD. Chest 2006;130:1096-101.

34. Estenne M, Maurer JR, Boehler A, et al. Bronchiolitis obliterans syndrome 2001: an update of the diagnostic criteria. J Heart Lung Transplant 2002;21:297-310.

35. Reid KR, McKenzie FN, Menkis AH, et al. Importance of chronic aspiration in recipients of heart-lung transplants. Lancet 1990;336:206-8.

36. Kirk AJB, Colquhoun IW, Corris PA, et al. Impaired gastrointestinal motility in pulmonary transplantation. Lancet 1990;336:752

37. Au J, Hawkins T, Venables C, et al. Upper gastrointestinal dysmotility in heart-lung transplant recipients. Ann Thorac Surg 1993:55:94-7.

38. Lubetkin EI, Lipsom DA, Palevsky HI, et al. Gl complications after orthotopic lung transplantation. Am J Gastroenterology 1996:91:2382-90.

39. Rinaldi M, Martinelli L, Volpato G, et al. Gastro-esophageal reflux as cause of obliterative bronchiolitis: a case report. Transplant Proc 1995;27:2006-7.

40. Cantu E 3rd, Appel JZ 3rd, Hartwig MG, et al. J. Maxwell Chamberlain Memoria Paper. Early fundoplication prevents chronic allograft dysfunction in patients with gastroesophageal reflux disease. Ann Thorac Surg 2004;78:1142-51.

41. Palmer SM, Miralles AP, Howell DN, et al. Gastroesophageal reflux as a reversible cause of allograft dysfunction after lung transplantation. Chest 2000;118:1214-17.

42. Hadjiliadis D, Duane Davis R, Steele MP, et al. Gastroesophageal reflux disease in lung transplant recipients. Clin Transplant 2003;17:363-8.

43. Tamhankar AP, Peters JH, Portale G, et al. Omeprazole does not reduce gastroesophageal reflux: new insights using multichannel intraluminal impedance technology. J Gastrointest Surg 2004;8:888-96.

44. Young LR, Hadjiliadis D, Davis RD, et al. Lung transplantation exacerbates gastroesophageal reflux disease. Chest 2003;124:1689-93.

45. D'Ovidio F, Mura M, Ridsdale R, et al. The effect of reflux and bile acid aspiration on the lung allograft and its surfactant and innate immunity molecules SP-A and SP-D. Am J Transplant 2006;6:1930-8.

46. Berkowitz N, Schulman LL, McGregor C, et al. Gastroparesis after lung transplantation. Potential role in postoperative respiratory complications. Chest 1995;108:1602-7

47. D'Ovidio F, Mura M, Tsang M, et al. Bile acid aspiration and the development of bronchiolitis obliterans after lung transplantation. J Thorac Cardiovasc Surg 2005; 129:1144-52.

48. D'Ovidio F, Keshavjee S. Gastroesophageal reflux and lung transplantation. Dis Esophagus 2006;19:315-20.

49. Gotley DC, Morgan AP, Ball D, et al. Composition of gastro-oesophageal refluxate. Gut 1991;32:1093-9.

50. Kauer WK, Peters JH, DeMeester TR, et al. Composition and concentration of bile acid reflux into the esophagus of patients with gastroesophageal reflux disease. Surgery 1997;122:874-81.

51. Kauer W, Peters JH, DeMeester T, et al. Mixed reflux of gastric and duodenal juices is more harmful to the esophagus than gastric juice alone. The need for surgical therapy re-emphasized. Ann Surg 1995;222:525-31.

52. Ward C, Forrest IA, Brownlee IA, et al. Pepsin like activity in bronchoalveolar lavage fluid is suggestive of gastric aspiration in lung allografts. Thorax 2005;60:872-4.

53. Farrell S, McMaster C, Gibson D, et al. Pepsin in bronchoalveolar lavage fluid: a specific and sensitive method of diagnosing gastroesophageal reflux-related pulmonary aspiration. J Pediatr Surg 2006;41:289-93.

54. Stovold R, Forrest IA, Corris PA, et al. Pepsin, a biomarker of gastric aspiration in lung allografts: a putative association with rejection. Am J Respir Crit Care Med 2007:175:1298-303.

55. Hartwig MG, Appel JZ, Li B, et al. Chronic aspiration of gastric fluid accelerates pulmonary allograft dysfunction in a rat model of lung transplantation. J Thorac Cardiovasc Surg 2006;131:209-17.

56. Theisen J, Nehra D, Citron D, et al. Suppression of gastric acid secretion in patients with gastroesophageal reflux disease results in gastric bacterial overgrowth and deconjugation of bile acids. J Gastrointest Surg 2000:4:50-4.

57. Heyland DK, Cook DJ, Schoenfeld PS, et al. The effect of acidified enteral feeds on gastric colonization in critically ill patients: results of a multicenter randomized trial. Canadian Critical Care Trials Group. Crit Care Med 1999:27:2399-406.

58. Gleeson K, Eggli DF, Maxwell SL. Quantitative aspiration during sleep in normal subjects. Chest 1997:111:1266-72.

59. Herve P, Silbert D, Cerrina J, et al. Impairment of bronchial mucociliary clearance in long-term survivors of heart/lung and double-lung transplantation. The Paris-Sud Lung Transplant Group. Chest 1993:103:59-63. 
60. Higenbottam $\mathbf{T}$, Jackson $M$, Rashdi $T$, et al. Lung rejection and bronchial hyperresponsiveness to methacholine and ultrasonically nebulized distilled water in heart-lung transplantation patients. Am Rev Respir Dis 1989:140:52-7.

61. Oelschlager BK, Chang L, Pope CE 2nd, et al. Typical GERD symptoms and esophageal $\mathrm{pH}$ monitoring are not enough to diagnose pharyngeal reflux. J Surg Res 2005; 128:55-60.

62. Potluri S, Friedenberg F, Parkman HP, et al. Comparison of a salivary/sputum pepsin assay with 24-hour esophageal $\mathrm{pH}$ monitoring for detection of gastric reflux into the proximal esophagus, oropharynx, and lung. Dig Dis Sci 2003;48:1813-17.

63. Bredenoord AJ, Weusten BL, Timmer R, et al. Characteristics of gastroesophageal reflux in symptomatic patients with and without excessive esophageal acid exposure. Am J Gastroenterol 2006;101:2470-5.

64. Bredenoord AJ, Tutuian R, Smout AJ, et al. Technology review: esophageal impedance monitoring. Am J Gastroenterol 2007;102:187-94.
65. Ravelli AM, Panarotto MB, Verdoni L, et al. Pulmonary aspiration shown by scintigraphy in gastroesophageal reflux-related respiratory disease. Chest 2006;130:1520-6.

66. Mainie I, Tutuian R, Shay S, et al. Acid and non-acid reflux in patients with persistent symptoms despite acid suppressive therapy: a multicentre study using combined ambulatory impedance-pH monitoring. Gut 2006;55:1398-402.

67. Tutuian R, Mainie I, Agrawal A, et al. Nonacid reflux in patients with chronic cough on acid-suppressive therapy. Chest 2006;130:386-91.

68. Hunt J, Yu Y, Burns J, et al. Identification of acid reflux cough using serial assays of exhaled breath condensate $\mathrm{pH}$. Cough 2006;2(1):3.

69. Dupont LJ, Dewandeleer Y, Vanaudenaerde BM, et al. The pH of exhaled breath condensate of patients with allograft rejection after lung transplantation. Am J Transplant 2006;6:1486-92.

70. Krishnan A, Chow S, Thomas P, et al. Exhaled breath condensate pepsin: A new noninvasive marker of GERD after lung transplantation. J Heart Lung Transplant 2007;26(Suppl 1):S139.

\section{Pulmonary puzzle}

\section{Answer}

From the question on page 100

Immunoassay for Treponema pallidum antibodies (TPAb ELISA) and the rapid plasma reagin test (RPR) performed at presentation were positive at high titres (1:16 and 1:128, respectively). TPAb were also detected in the cerebrospinal fluid (titre 1:128). T pallidum DNA-PCR of lung biopsy was not performed because of the poor specimen available.

Following the clinical and serological diagnosis of secondary syphilis with involvement of the central nervous system, intravenous ceftriaxone ( $2 \mathrm{~g}$ /day) was administered for 2 weeks because the patient reported allergy to penicillin. An unexpected and significant reduction in the major lung lesion was observed at the end of the antibiotic therapy and complete radiological disappearance of all pulmonary lesions had occurred at 3-month follow-up. TPAb and RPR titres both decreased to 1:8 after 3 months and RPR was negative at 6 months.

$\mathrm{HIV} /$ syphilis co-infection is associated with high rates of asymptomatic primary syphilis and with atypical features of secondary disease at presentation. ${ }^{1}$ Lung involvement is extremely rare during secondary syphilis and it has been described mainly in patients with tertiary stage of the disease.

David and colleagues ${ }^{2}$ recently reviewed nine cases published since 1967 which met the Coleman criteria for the diagnosis of secondary pulmonary syphilis (ie, physical findings of secondary syphilis, serological diagnosis, radiological lung abnormalities, exclusion of other forms of pulmonary disease and resolution of radiological abnormalities following anti-syphilis treatment). ${ }^{3}$ Interestingly, eight of the nine patients had single or multiple lesions at lower lung regions. ${ }^{2}$ Our patient with diagnosed secondary syphilis also had bibasilar lesions and alternative possible aetiologies for pulmonary lesions were excluded, both microbiologically and histologically. Even in the past when tertiary syphilis was not uncommon, one criterion to discriminate pulmonary syphilis from tuberculosis was the tendency of the former to attack the middle and lower lobes. ${ }^{4}$ One explanation for the prevalent involvement of the lower lobes during pulmonary syphilis may be ascribed to the high oxygen sensitivity of the mircoorganism due to the absence of microbial enzymes that detoxify reactive oxygen species. ${ }^{5}$ Despite its ability to spread in any tissue, $T$ pallidum thus encounters an unfavourable environment in the lung. When pulmonary involvement occurs, it will conceivably take place in the less oxygenated area of the organ. The differential diagnosis for pulmonary nodules associated with cutaneous lesions includes a broad range of diseases (lymphoma, Kaposi sarcoma, metastatic malignancies, Wegener granulomatosis, sarcoidosis, mycobacteriosis, disseminated fungal infections and septic emboli); syphilis should also be added to the list.

Acknowledgements: The authors thank Dr M Nebuloni, Chair of Pathology, L Sacco Hospital, University of Milan for her helpful contribution to the histological analysis and Dr M Corbellino, Department of Clinical Sciences, Luigi Sacco Hospital, Section of Infectious Diseases and Immunopathology, University of Milan for help with editing.

Thorax 2009;64:173. doi:10.1136/thx.2007.098178a

\section{REFERENCES}

1. Lynn WA, Lightman S. Syphilis and HIV: a dangerous combination. Lancet Infect Dis 2004;4:456-66.

2. David G, Perpoint T, Boibieux A, et al. Secondary pulmonary syphilis: report of a likely case and literature review. Clin Infect Dis 2006:42:e11-15.

3. Coleman DL, McPhee SJ, Ross TF, et al. Secondary syphilis with pulmonary involvement. West J Med 1983;138:875-8.

4. Morgan AD, Lloyd WE, Prince-Thomas C. Tertiary syphilis of the lung and its diagnosis. Thorax 1952;7:125-33.

5. LaFond RE, Lukehart SA. Biological basis for syphilis. Clin Microbiol Rev 2006;19:29-49. 